\title{
Optimized Bi-Connected Federation of Multiple Sensor Network Segments
}

\author{
Sookyoung Lee ${ }^{1}$, Mohamed Younis ${ }^{2}$ and Meejeong Lee ${ }^{1}$ \\ ${ }^{I}$ Department of Computer Science and Engineering, Ewha Womans University, Seoul, Korea (sookyoung,lmj@ewha.ac.kr) \\ ${ }^{2}$ Department of Computer Science and Electrical Engineering, University of Maryland, Baltimore County,
}

Baltimore,MD,USA (younis@umbc.edu)

\begin{abstract}
Wireless sensor networks (WSNs) serving in hostile environments are susceptible to simultaneous failure of multiple collocated nodes that cause the network to split into distinct segments. Restoring connectivity would be needed to resume full WSN operation. Similar scenario is encountered when autonomous WSNs have to collaborate for achieving a common task. Proactively provisioning spare connections would be also desirable to prevent possible partitioning caused by a single node failure in the federated topology. In this paper, we present an optimized strategy for establishing 2-vertex distinct paths between every pair of segments or individual WSNs while minimizing the number of the deployed relay nodes (RNs) and increasing the efficiency of the resulting biconnected topology in terms of average node degree. The proposed Optimized Bi-Connected federation of multiple sensor network segments $(\mathrm{OBiC})$ algorithm strives to form a single simple cycle which visits every segment exactly once. The least number of relays are populated along the steinerized edges of the formed bi-connected topology by the cycle and thus 2-vertex inter-segment disjoint paths are guaranteed. We analyze the properties of $\mathrm{OBiC}$ mathematically and validate its performance through extensive simulation experiments. The validation results show that $\mathrm{OBiC}$ yields highlyconnected topologies with short inter-segment paths while employing fewer RNs than competing schemes.
\end{abstract}

Keywords-Wireless sensor networks, Network Partitioning, Topology Repair, 2-vertex Connectivity, Fault Tolerance, Relay Node Placement

\section{INTRODUCTION}

WSNs have attracted growing interest in recent years due to their numerous applications, especially those serving in harsh environments [1]. In a hostile application setup, such as coast and border surveillance, search-and-rescue and battlefield reconnaissance, employing a WSN that operates unattended would decrease the cost of the application and avoid the risk to human life. Since a sensor node is typically miniaturized and constrained in its energy, computation and communication resources, a large set of sensors are deployed to ensure area coverage and increase the fidelity of the collected data [2]. The sensor nodes are expected to stay reachable to each other in order to coordinate their actions while performing a task, and to forward their readings to in-situ users. Therefore, the inter-sensor connectivity has a significant impact on the effectiveness of WSNs and should be sustained all the time.

Meanwhile, a sensor is susceptible to failure due to the small form factor and limited onboard energy supply. Such a failure may be limited to a single node that is caused by battery depletion, or due to malfunction or external hazard. If the faulty node is critical, i.e., serve as a cut-vertex in the WSN topology, the network may get partitioned into disjoint segments [5]. Such partitioning can be avoided by provisioning bi-connectivity at the time of network setup. Moreover, WSNs operating in a harsh environment may suffer from a major damage that results in simultaneous failure of multiple collocated nodes causing the network to split into disjoint segments. For example in a battlefield, parts of the deployment area may be attacked by explosives and some nodes get destroyed. Figure 1-(a) shows an articulation, where the dark circles represent the damaged nodes and the surviving sensor nodes, denoted by light circles, are grouped into seven disjoint partitioned WSNs due to the loss of connectivity. This type of failure is handled by relay node placement to reestablish a connected inter-segment topology after the failure takes place. Mitigating the simultaneous failure of multiple collocated nodes through resource provisioning at the time of network setup would require massive resources that will most probably involve nodes outside the area of deployment due to susceptibility to damage. Thus, it is not cost effective to guard the network against such type of failure.

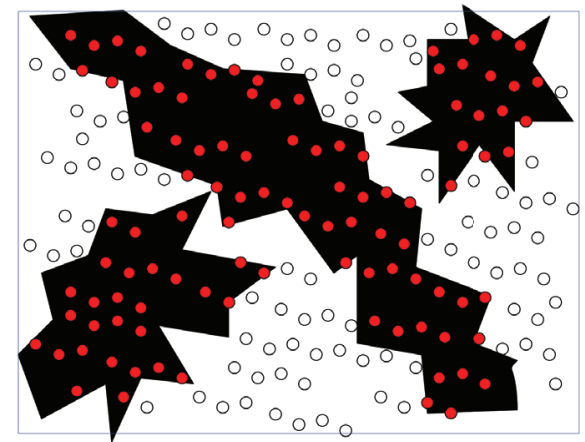

(a)

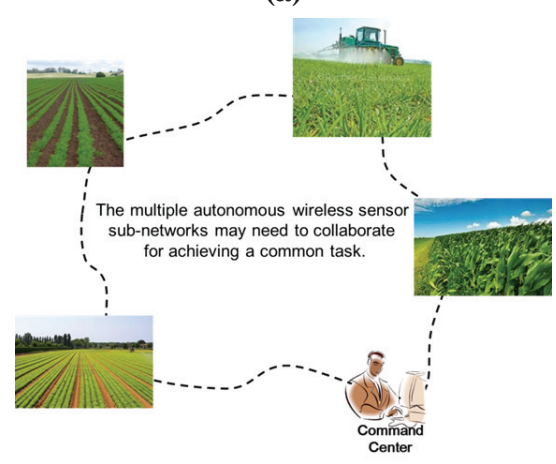

(b)

Figure 1. Illustration of two scenarios which require inter-segment connectivity in WSNs, (a) a partitioned WSN due to multiple failure of collocated sensors (b) standalone WSNs are to be federated in order to achieve a common task, e.g., set pressure of water sprinklers. 
The focus on this paper is on recovery from the simultaneous failure of multiple collocated nodes with an additional objective of tolerating future single node failures in the recovered network topology. The rationale for such an objective is that shortly after the recovery a node may be damaged due to aftermath or unexploded bombs, etc. In addition, the probability of a single node failure is generally higher than that of multiple collocated nodes, due to causes such as exhaustion of the onboard energy supply and electronic breakdown, as mentioned above. Bi-connecting the formed inter-segment topology will help in reducing the probability of network re-partitioning after recovery. In addition to WSN partitioning caused by node failure, another scenario is considered in the paper where multiple autonomous WSN segments are required to collaborate to achieve a common task as seen in Figure 1-(b). Forming a biconnected inter-segment topology, i.e., establishing two vertex-disjoint paths among every pair of segments would boost the application robustness and balance the inter-segment traffic in the network.

We shall use the term federation to refer to establishing connectivity in both scenarios, i.e., repairing a damaged WSN or linking multiple independent WSN segments. Federating multiple WSN segments while forming a bi-connected topology is an under-researched problem. When the network nodes are not mobile, the topology cannot be autonomously reconfigured and relay nodes (RNs) need to be deployed to achieve the fedeartion goal. Naturally the RN count opts to be minimized in order to reduce the federation overhead. Therefore, the federation problem tackled in the paper is fundamentally how to establish a 2-vertex connected intersegment topology by deploying the least RN count. Such RN placement optimization problem can be mapped to forming Steiner Tree with minimum Steiner points and Bounded EdgeLength, which is proven to be an NP-hard problem by Lin and Xue [3]. In order to address such complexity this paper presents $\mathrm{OBiC}$, a polynomial-time $\underline{\text { Optimized Bi-Connected }}$ federation of multiple sensor network segments. In addition to the 2-vertex connectivity, $\mathrm{OBiC}$ also opts to boost the average node degree in order to further provide route alternatives and enable load balancing.

In order to meet the bi-connectivity goal with the least cost, $\mathrm{OBiC}$ strives to form a single simple cycle of all segments which is a Hamiltonian cycle. A Hamiltonian cycle of a graph allows no repetition of vertices (segments) other than the repetition of the starting and ending one. Finding a Hamiltonian cycle is a known NP-Hard problem [4]. Therefore, OBiC pursues a heuristic approach that is composed of three phases. During the first phase, OBiC constructs a digraph $d G=(d V, d E)$ where each segment is connected to the nearest neighbor inward toward the center of the area and also linked to at least one outer segment. For identifying the inter-segment inward edges, OBiC groups segments as tiered border terminals found by a nested set of convex hulls of the segments. Based on the found $d G$, in the $2^{\text {nd }}$ phase OBiC strives to identify a concave hull of the segments, denoted by Cave. Then OBiC opts to bi-connect the segments inside Cave using the found inward edges in $d E$, which results in more distorted concave hull with its size minimized. Throughout the minimization of Cave, $\mathrm{OBiC}$ eventually forms a Hamiltonian cycle, represented as an undirected graph $G_{2 c}=\left(V_{2 c}, E_{2 c}\right)$. During the $3^{\text {rd }}$ phase, OBiC optimizes the identified $G_{2 c}$ by reducing the total length of the circumference and finally populates the least count of RNs on each edge $e \in E_{2 c}$ to connect the two end segments of $e$.

The rest of the paper is organized as follows. Related work is covered in Section II. The problem is formally defined and the considered system model is described in Section III. The details of $\mathrm{OBiC}$ are provided in Section IV. The validation results are presented in Section V. The paper is finally concluded in Section VI.

\section{RELATED WORK}

Published techniques for tolerating node failures employ precautionary or reactive methodologies [5][6]. The precautionary methodology proactively provisions spare connections in the network at the time of deployment or during normal operation so that the network does not suffer from partitioning due to the failure of a single or multiple nodes. In order to form a WSN topology which is resilient to $k$-1 node failures, the proactive fault-migration strategy strives to establish $k$-node distinct communication paths between every pair of nodes in the network. Employing the least number of redundant nodes to achieve such fault resilience is usually a design objective for approaches that follow the precautionary methodology [7][8]. On the other hand, the reactive methodology opts to repair the network topology and restore the WSN connectivity to its pre-failure level. Algorithms in this category, either utilize mobility of healthy nodes to restore connectivity [9][10], or employ additional nodes during recovery [11][12].

$\mathrm{OBiC}$ is a connectivity restoration algorithm which strives to deploy the least count of additional RNs to repair a partitioned network into a 2-connected topology. OBiC can also be applied proactively if a set of autonomous WSNs are to be federated by forming a robust topology that can tolerate an occasional single $\mathrm{RN}$ failure. Given the scope of the contribution, in the balance of this section we focus on published work in the proactive category for which $k$ connected topology is established. For coverage of reactive algorithms and techniques for forming 1-connected topology, the reader is referred to [5][6]. In addition, OBiC is set apart from contemporary algorithms for finding a Hamiltonian cycle in a graph.

Bi-connectivity: Hao et al. [7] strive to place the fewest RNs such that each sensor is connected to at least two RNs and the inter-RN network is bi-connected. The authors formulate the placement problem as "2-Connected Relay Node Double Cover", to determine the locations of the fewest RNs, so that each sensor is covered by the communication range of at least two RNs and the group of RNs become bi-connected. All RNs 
are assumed to have the same communication range $R$ that is at least twice the range of a sensor node $r$. The proposed algorithm computes a possible position $p$ of a relay and a set of sensor nodes $C(p)$ which is covered by a relay placed at $p$. These positions can be found at the intersections of the communication ranges of neighboring sensors. Then, the algorithm identifies the positions at which RNs are placed to cover the maximum number of sensors. The algorithm checks whether the RNs form a 2-connected graph and every sensor can reach at least 2 RNs. If not, more relays are populated. The latter step is repeated till the objective is achieved. Tang et al. [8] pursues a similar idea while modeling the area as a grid. The connectivity is first handled within the individual cells and then at the inter-cell level. However, $R$ in this case is assumed to be at least four times as much as $r$. Unlike OBiC, these approaches do not consider the scenario where the intersensor distance is more than $R$ which necessitates the formation of multi-hop inter-RN paths.

In addition, a polynomial time heuristic to achieve 2connectivity among $n$ nodes with the least relay count assuming $R=r$ is presented by Kashyap et al.[13]. The authors focus on establishing both $k$-edge and $k$-vertex connectivity separately by using the algorithm in [14]. In order to form the $k$-vertex connectivity, they first compute $k$-vertex connected spanning sub-graph $g$ and then steinerize each edge of $g$. This is the best known heuristic in the literature for minimizing the relay count for bi-connectivity, i.e., $k=2$, and effectively forms a large ring that includes all segments by considering local proximity from each segment. $\mathrm{OBiC}$ forms a more efficiently connected network in terms of the node-degree of the intersegment topology and minimizes the count of relays by factoring in the inter-segment distance toward the center of the area of segments. In other words, OBiC forms a spanning subgraph with inward inter-segment edges. The performance of $\mathrm{OBiC}$ is compared to this heuristic through simulation as discussed in Section 5. Meanwhile, Zhang et al. [15] study two variants of the problem, namely, single-tiered and two-tiered relay placement for $R \geq r$. In the single-tier version, a path between two sensors, segments in the context of OBiC, may have both RNs and sensors. In the two-tier version, only RNs can serve on the paths between terminals. The proposed algorithm for the single-tier version is in essence a generalization of the approach of [13] for $R>r$. The two-tier solution steinerizes the edges of minimum 2-connected spanning sub-graph using [14] in a similar way to that of [13].

The 2C-SpiderWeb approach [16] establishes an intersegment topology that resembles a spider web, for which the segments are situated at the perimeter and a mesh is formed at the center of the deployment area. An edge between each terminal and the centroid is formed and RNs are placed at a distance $R$ from the centroid. The edge between each pair of $\mathrm{RNs}$ on the mesh is then steinerized. To achieve 2-vertex connectivity each terminal at the perimeter is connected to two distinct RNs on the inner mesh through $2 \mathrm{RN}$-disjoint paths. In other words, 2C-SpiderWeb bi-connects segments based on the topology that grows toward the center from the perimeter of the formed polygon. Moreover, CRAFT [17] strives to restore the partitioned network into a bi-connected topology by forming an inner ring based on the location of the inmost terminals. CRAFT then identifies Steiner Points (SPs) to connect outer terminals to the inner ring with the least cost. The other terminals are bi-connected by establishing 2-vertex disjoint paths through the inner ring using the identified SPs. Unlike 2C-SpiderWeb and CRAFT, OBiC establishes biconnectivity by forming a Hamiltonian cycle which visits every segment only once based on the inter-segment paths from the perimeter toward the center of the area. The RN count and topology efficiency of $\mathrm{OBiC}$ are compared to those of 2C-SpiderWeb and CRAFT in Section 5.

K-connectivity: Some of the published solutions have focused on the general $k$-connectivity problem. For example, a graphtheory based solution is proposed in [18]. First, a complete graph, say $G$, for the set of vertices (nodes) is formed and each added edge is associated with a weight based on the number of required RNs on the edge $e(u, v)$ to establish connectivity between $u$ and $v$. The problem is then mapped to finding a minimum-weight $k$-vertex-connected sub-graph " $g$ ". Finally, missing links (edges) in $g$ are established by deploying additional nodes. A similar idea is pursued in [19] for a heterogeneous WSN in terms of the transmission range of the network nodes. Meanwhile, in [20][21][22] the authors assume that all RNs have the same communication range and solve the "minimum k-vertex connected spanning graph" (MKCSG) problem. Then the algorithm places the least number of RNs to establish $k$-vertex connectivity $(k \geq 1)$ by providing a one-way or a two-way steinerized path along each edge of the found MKCSG. Moreover, in [23] $k$-connectivity is provided as a byproduct of determining a connected dominating set to obtain a robust backbone.

Furthermore, $k$-connectivity has been also studied along with the coverage problem under different sensor transmission $(r)$ and sensing $(s)$ ranges. Typically, $r>s$ in many applications and thus once full coverage is achieved the network topology often becomes strongly connected. It is shown in [24] that for a grid structure the network will be connected as long as $r>3 s$. The results have been extended to higher level of connectivity by proposing to use some deployment patterns. For instance, in [25], the authors prove that a strip pattern of sensors can provide optimal full coverage along with 2-connectivity for all different $r / s$ ratios. The horizontal strips are formed by a distance of $\alpha=\min \{r$, $3 s\}$ while the vertical strips are formed by a distance of $\beta=s+s 2-\alpha 2 / 4$. In addition, horizontal strips are shifted to the right a distance of $\alpha 2$ at alternating rows to guarantee 2connectivity among the nodes. The work has been extended to $3,4,5$, and 6 connectivity using different deployment patterns [26][27]. Obviously these approaches are limited to WSNs for which $r>\sqrt{ } 3 s$. Wang et al., [28] prove that $k$-coverage implies $k$-connectivity of the entire network in WSNs if $r>2 s$. 
Meanwhile, the focus of the approach in [29] is on 3D setups with the additional objective of prolonging the network lifetime while making the network resilient to up to $k$ independent node failures. A grid model is employed in which nodes are allowed to be positioned at the intersection points. The authors formulate a node relocation problem for balancing the traffic load and thus extending the network longevity as a mixed integer linear program optimization. A variant of the approach is presented in [30] where the objective is to minimize the number of involved RNs, while ensuring a lower bound on the network lifetime. In addition, the approaches of [31][32] also have the same objectives of expanding the network lifetime by minimizing power consumption while forming $k$-connectivity topology. Three mixed integer programs are formulated by considering four variants of the optimization problem depending on the link symmetry and directionality, i.e., unidirectional, of bidirectional in the topology [31]. Meanwhile, the approach in [32] addresses the $k$-degree anycast topology control problem. It employs a super-node with unlimited energy resources and opts to adjust a transmission range of each sensor such that $k$-vertex-disjoint paths to super-nodes are formed and the total power consumption is minimized. Although the $k$-connectivity approaches $(k>2)$ generate more resilient topology than that of the bi-connectivity algorithms, the topology formation may be too costly and impractical as $k$ grows.

Hamiltonian cycle: A number of studies have considered the formation of a Hamiltonian cycle (HC) in a graph [33-36]. The approaches of [33][34] strive to find a $\mathrm{HC}$ in a tournament which is a directed graph $T=(V, E)$ where for every pair of vertices, $u$ and $v$, exactly one of $(u, v),(v, u)$ belongs to $E$. In [33] a parallel algorithm is proposed for finding a restricted Hamiltonian path in which the first or the last vertex of the path is fixed. Meanwhile, the algorithm of [34] opts to find a HC in $T$ with $n$ vertices if any exists. The main objective is to reduce the searching complexity to $\mathrm{O}(m)$, where $m=n(n-1) / 2$ from those of the previous ones e.g., $\mathrm{O}\left(n^{3}\right)$ or $\mathrm{O}\left(n^{2} \log n\right)$. On the other hand, the focus in [35][36] is on identifying a $\mathrm{HC}$ in a random graph. The main focus of [35] is on showing that the $\mathrm{HC}$ problem is well-solved in a probabilistic sense and providing a polynomial time algorithm to search for Hamiltonian cycles in an undirected random graph. A more complicated random graph is considered in [36] in which there are $n$ edges forming a specific $\mathrm{HC}$ and $d n$ random edges hiding the cycle. Then an $\mathrm{O}\left(n^{3} \log n\right)$ steps algorithm is proposed to find a hidden $\mathrm{HC}$.

Overall, these algorithms mainly strive to find a $\mathrm{HC}$ in a given input graph with various conditions without considering the efficiency of the formed topology in terms of average node degree or a size of the formed cycle. In other words, the property of a wireless link such as a communication range is not considered while forming a $\mathrm{HC}$ in the approaches. $\mathrm{OBiC}$ opt to form an efficient $\mathrm{HC}$ which visits all segments once considering a radio range of a $\mathrm{RN}$ and reduces a total $\mathrm{RN}$ count to form the cycle.

\section{PROBLEM STATEMENT}

In this paper two scenarios are considered; a set of standalone networks or disjoint segments of a single WSN which was subject to a major damage, e.g., inflicted by explosives in a battlefield or landslides/avalanches. Our objective is to establish a cut-vertex free topology which is tolerant to a single node failure by providing 2-vertex disjoint paths between every pair of partitions using the least number of relay nodes. A cut-vertex is the node in a graph whose removal causes the graph to partition into disjoint sub-graphs. It is assumed that each segment is represented by a terminal, e.g., a gateway node that serves as an interface for the segment. Such a terminal node can be specified by a network operator in case the segment is a standalone WSN. When considering partitions of a damaged topology, designating the terminal can be incorporated in the failure detection procedure. An example procedure is as follows.

Upon failure of a set of collocated nodes, their 1-hop neighbors notice the node loss and assess the scope by correlating consecutive node failures in the vicinity, e.g., by witnessing a major and sudden drop in communication traffic and/or by identifying unreachability of a certain set of remote nodes. Upon confirming the multi-node failure and the split of the network into disjoint segments, these neighbors become the border nodes and broadcast a message on active links to notify all reachable nodes, which will naturally belong to their segment. After some pre-determined convergence time a representative terminal (a gateway) for each segment is elected in regard to a variety of terminal selection attributes such as application features, onboard energy, a degree of connectivity, etc. Due to the application dependency of the terminal selection criteria, the rest of the paper focuses more on forming the connected topology among the representatives rather than investigating the effect of the various attributes. Figure 2-(a) which serves as a working example in this paper, shows the terminals, $\mathrm{Seg}_{1}, \mathrm{Seg}_{2}, \ldots, \mathrm{Seg}_{27}$, that representing the individual segments.

Thus, the problem that we tackle in this paper becomes forming a 2-connected topology among the terminals $\mathrm{Seg}_{i}$ 's by populating the least number of additional RNs such that 2vertex disjoint paths between every pair of segments $\mathrm{Seg}_{c}$ and $\mathrm{Seg}_{d}$ are provided, which eventually forms a simple cycle between them, and a network (re)partitioning caused by a single $R N$ failure is thus prevented. The number of relays $N_{R N}$ is captured mathematically by the following formula:

$$
\begin{gathered}
N R N=\operatorname{argmin} N(i) i=O N \operatorname{seg}-1 j=O N \operatorname{seg}-1, j \neq i S C S e g c, \\
S e g d,
\end{gathered}
$$

where Nseg is the number of segments,

$$
\begin{aligned}
& \text { SCSegs, Segd }=\quad \begin{array}{c}
R N k, k=0, . ., N i-1 \\
R N l \neq R N m, \forall l, m \in\{0, . ., N i-1\} \text { Segs } \neq \text { Segd } \in R N k
\end{array}
\end{aligned}
$$


In formula (1), a simple cycle between $\mathrm{Seg}_{s}$ and $\mathrm{Seg}_{d}$ denoted as $S C\left(\operatorname{Seg}_{s}, \operatorname{Seg}_{d}\right)$ is represented as a list of RNs and $\mathrm{Seg}_{i}$ 's, $i \neq s$ and $d$ in which two vertex-disjoint paths between $S_{e} g_{s}$ and $\operatorname{Seg}_{d}$ with no intermediate repeated. Since the formulated problem is known NP-hard [3], we pursue heuristics.

The paper focuses on the algorithmic aspect of the internetworking problem without considering the diversity of the physical, link and network layers. Moreover, the deployed RNs are assumed to be more capable than a sensor node, with significantly richer energy reserve, and stronger computation power. It is also assumed that all relays have the same communication range $R$ which is equal to that of a sensor $r$, i.e., $R=r$. This is a simplifying assumption to ease the presentation; the approach can handle the case when the RN has larger communication range than a sensor node. In addition, coverage is not the focus of $\mathrm{OBiC}$ although $\mathrm{RNs}$ may have sensing capabilities that can mitigate coverage loss caused by the damage. Intuitively relays are more expensive than stationary sensors, and the number of deployed relays is thus naturally fewer and should be minimized during the biconnectivity establishment process.

\section{IV.The OBiC APPROACH}

To federate segments with the provision of 2-vertex disjoint paths, OBiC basically strives to form a ring topology for all segments where the inner area is minimized. In order to achieve such an optimized ring topology, $\mathrm{OBiC}$ first tries to identify the boundaries of the area covered by all segments. There are two methodologies to compute the boundaries of a given set of points such as a convex hull and a concave hull. Since a convex hull does not represent well the boundaries when the number of the involved points is large and the points may form trees, in this paper we opt to identify the concave hull of the segments, denoted hereafter as Cave. The Cave is computed based on the convex hull of all segments and thus contains some segments inside. Then OBiC bi-connects the internal segments to the ones on the boundary of Cave to form a ring topology where every segment lies on the ring. For computing Cave and bi-connecting the internal segments, $\mathrm{OBiC}$ first identifies the acyclic digraph $d G$ which includes the least cost directed inter-segment edges toward the center of the area with no directed cycle. Thus, OBiC consists of three phases: (1) categorizing the segments based on the proximity from the boundary of the area of interest and identifying the $d G$, (2) computing the Cave, and forming a bi-connected undirected graph $G_{2 c}=\left(V_{2 c}, E_{2 c}\right)$ by connecting internal segments via Cave, and (3) optimizing $G_{2 c}$ by raveling the edges in $E_{2 c}$ and finally populating the least count of relays on each edge in $E_{2 c}$. The details of $\mathrm{OBiC}$ are provided in the balance of this section. We note that a list of the acronyms used is provided in Appendix A for a quick reference.

\section{A. $1^{\text {st }}$ Phase: Identifying an acyclic digraph $d G$ inward toward the center of the area}

During the $1^{\text {st }}$ phase OBiC first (i) categorizes the segments in rounds by identifying nested convex hulls ( $\mathrm{CHs}$ ) based on which the segments are split in terms of their relative proximity to the periphery of the area of interest. Thus, the segments lying on each $\mathrm{CH}$ are grouped. Then, (ii) a digraph $d G$ is formed by finding directed inter-segment edges between adjacent groups. These steps are explained in detail below.

Categorizing segments: The categorization of a set of segments $S_{\text {seg }}$ is performed in rounds. In the first round, OBiC defines the polygon which covers all segments by computing the convex hull of the segments using Graham scan algorithm [37]. The algorithm identifies the border of $\left|S_{\text {seg }}\right|$ segments by starting from the left-most (least y-coordinate) segment $\mathrm{Seg}_{L}$, then sorting segments in increasing order of an angle with $x-$ axis relative to $\operatorname{Seg}_{L}$ and finally selecting segments from the sorted list so that right-turns are maintained when navigating from $\operatorname{Seg}_{L}$ until ending at $\operatorname{Seg}_{L}$. We denote the convex hull found in the first round $r=1$ as $\mathrm{CH}_{0}$ and the segment lying on $\mathrm{CH}_{0}$ as the outmost segments of the first round, in short $\mathrm{OS}^{0}$.

In the second round $(r=2), \mathrm{OBiC}$ continues to form the convex hull of segments inside $\mathrm{CH}_{0}$, i.e., those in the set $\left(S_{\text {seg }}\right.$ $\left.-O S^{0}\right)$, to determine the second outmost segments $O S^{l}$. In the subsequent rounds $r$, the same procedure is repeated and the set $O S^{r-1}$ is thus found. The procedure terminates when the number of segments inside $\mathrm{CH}_{r-l}$ is less than three and thus no more convex hull can be formed. Therefore, $O S^{f-1}$ in the final round $f$ may form a polygon if $\left|O S^{f-1}\right| \geq 3$, a line which connects two remaining segments or a point in the case of only one segment left for round $f$. Due to the natural property of a convex hull, the polygon bounded by $\mathrm{CH}_{r-1}$ in round $r$ contains all $\mathrm{CH}_{k}$ 's of the subsequent rounds $k>r$. In other words, a set of the grouped segments in $O S^{k}$ is completely included by all $O S^{j}, 0<\forall j<k<f$-1. In Figure 2, $S_{\text {seg }}$ seen in Figure 2-(a) is split into four groups as seen in Figure 2-(b) which evidently shows the nested property of $O S^{j} \forall j$, i.e., $O S^{3}=\left\{\operatorname{Seg}_{10}, \operatorname{Seg}_{12}\right.$, $\left.\mathrm{Seg}_{14}, \mathrm{Seg}_{15}, \mathrm{Seg}_{16}\right\}$ lies inside $O S^{2}=\left\{\mathrm{Seg}_{11}, \mathrm{Seg}_{13}, \mathrm{Seg}_{21}, \mathrm{Seg}_{18}\right.$, $\left.\mathrm{Seg}_{17}, \mathrm{Seg}_{25}, \mathrm{Seg}_{9}, \mathrm{Seg}_{23}\right\}$, which is inside $\mathrm{OS}^{l}=\left\{\mathrm{Seg}_{7}, \mathrm{Seg}_{12}\right.$, $\left.\mathrm{Seg}_{6}, \mathrm{Seg}_{22}, \mathrm{Seg}_{20}, \mathrm{Seg}_{19}, \mathrm{Seg}_{5}, \mathrm{Seg}_{7}, \mathrm{Seg}_{8}, \mathrm{Seg}_{24}\right\}$, and all are contained by the polygon defined by $\mathrm{OS}^{0}=\left\{\mathrm{Seg}_{1}, \mathrm{Seg}_{2}, \mathrm{Seg}_{3}\right.$, $\left.\mathrm{Seg}_{4}\right\}$.

The rationale of grouping $S_{\text {seg }}$ into $O S s$ based on the nested convex hulls is that $\mathrm{OBiC}$ opts to find the Hamiltonian cycle of $S_{\text {seg }}$ via which each segment is connected to its nearest neighboring segments inward toward the core of the area. Between the adjacent convex hulls, each segment can identify its closest inward neighboring segment. Then based on the categorization, the acyclic digraph $d G=(d V, d E)$ will be identified later to include the shortest inter-segment edge from an outer OS $O S^{r-1}$ to next inner OS $O S^{r}, 0<r<f$-1, where $f$ is a final round. During the categorization of $S_{\text {seg }}$, two special cases are separately handled based on the layout of $S_{\text {seg: }}$ (i) If all segments in $S_{\text {seg }}$ belong to $O S^{0}$, OBiC steinerizes each edge of $O S^{0}$ considering a communication range of a relay and then 


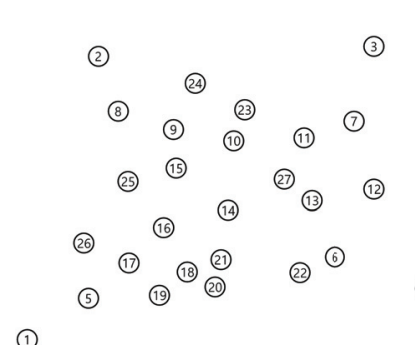

(a)

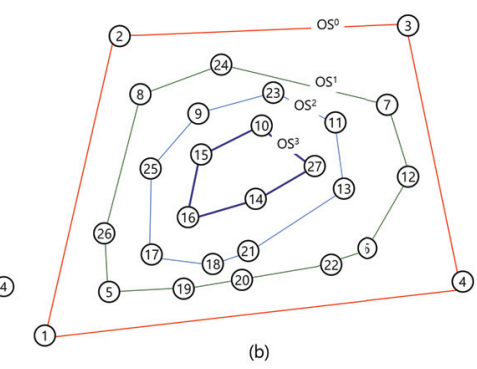

(b)
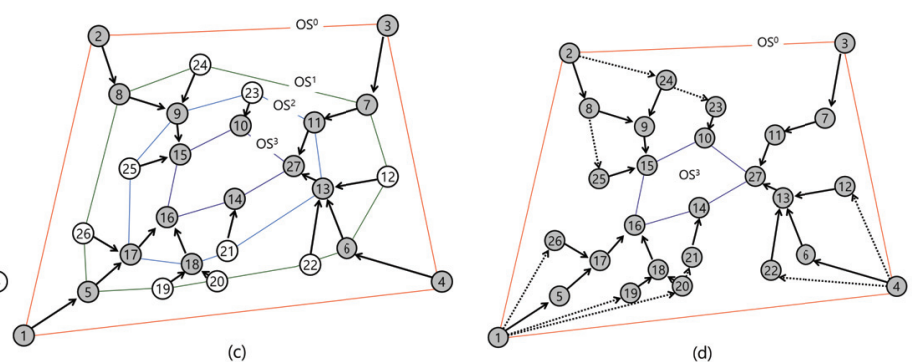

(d)

Figure 2. (a) shows layout of an example topology, $\left|S_{\text {seg }}\right|=27$, and (b) shows the result of the categorization of $S_{\text {seg. }}$. Through four rounds, $O S^{i}$, $\mathrm{i}=0,1,2,3$ are identified by convex hulls, based on which OBiC forms the acyclic digraph $d G$ shown in (c). For the white node that has only an out-going edge, an extra directed edge (marked as a dotted line) is added in $d E$ as seen in (d).

the segments are bi-connected by populating RNs at the found SPs; (ii) If all segments are collinear, and no polygon is thus identified in the first round albeit $\left|S_{\text {seg }}\right| \geq 3$, then OBiC steinerizes the edge " $e$ " between each pair of adjacent segments and populates double of the least count of RNs. Otherwise, OBiC tries to form a digraph $d G$ by adding inward inter-segment links between adjacent $O S$ s, as explained next.

Forming an acyclic digraph $d G$ : Based on the identified $O S$, OBiC tries to identify an acyclic directed graph $d G=(d V, d E)$, where $d V$ is $S_{\text {seg }}$ and $d E$ contains the inward directed edges from an outer group $O S^{r}$ to an inner one $O S^{r+1}$. Thus for each segment $N_{i} \in d V$ that belongs to $O S^{r}$, denoted hereafter as $N i r$, OBiC adds a directed edge $e(N i r, M j r+1)$ in $d E$ where $M j r+1$ is the nearest inner segment in $O S^{r+1}$. If there exist multiple $M j r+1$ 's that are away from Nir in the same distance, then the one which is located relatively on right side from Niris selected, as seen in an example of Figure 3. This condition helps in expediting the formation of Cave later.

As explained earlier, the rationale for finding the directed edges based on $O S$ s Figure 3. Special is that throughout a linked list of edges in case in forming $d G$ $d E$, OBiC tries to identify the vertex-

distinct path that starts from a node in $O S^{h}, 0<h<f$-2 and finally reaches at a node in $O S^{f-1}$ by visiting at most one node in each $O S^{x}, h<x<f-1$. For example in Figure 2-(c), $N 10$ and $N 241$ can reach at $O S^{3}$ via $\{e(N 10, N 51), e(N 51$, $N 172), e(N 172, N 163)\}$ and $\{e(N 241, N 92), e(N 92$, $N 153)\}$ respectively. In addition, some paths for $N 191$ and $N 201$ are merged at an intermediate node like $N 182$. After establishing the inward directed inter-OS edges, each node in $d V$ is classified into one of two types as seen in Figure 2-(c), a white node that has only an outgoing edge or a shaded node that has also an incoming edge as well. Since OBiC opts to finally form a ring topology among nodes in $d V$ based on which every node is visited only once, it is essential to ensure that there is a path reaching at a white node from the border of the area, i.e., the outmost polygon $\mathrm{CH}_{0}$. In other words, every node necessarily becomes an intermediate node at any path from the outmost group $O S^{0}$ to the inmost group $O S^{f-1}$. Therefore, for each white node $\mathrm{Nir}$ in $O S^{r} \mathrm{OBiC}$ adds an extra directed edge in $d E$, that comes from the nearest outer node $N h r-1$ in $O S^{r-1}$ going into Nir. The dotted edges in Figure 2(d) show the additional edges $e(N h r-1, N i r)$ for the white nodes.

For example in Figure 2-(d), for white nodes N241 and $N 232, e(N 20, N 241)$ and $e(N 241, N 232)$ are included in $d E$, respectively. In addition, for nodes $N 71, N 191$ and N202, three edges, each of which starts from the same node $N 10$ and ends at each of them, i.e., $e(N 20, N 241), e(N 20$, $N 241)$ and $e(N 241, N 232)$ are added. Therefore, $d E$ becomes $\{r=0 \ldots f-1 \mid e(N i r, M j r+1) \cup e(N h r-1, N i r)\}$, as seen in Figure 2-(d) as solid and dotted arrows, respectively. During the $d G$ formation, there are two notable cases depending on $\left|O S^{f-1}\right|$. If $\left|O S^{f-1}\right|$ equals one, every node in $O S^{f-2}$ is linked to the only node in $O S^{f-1}$. In addition, for the case $\left|O S^{f-1}\right|=2$, i.e., $O S^{f-1}$ forms a line $l\left(e_{1}, e_{2}\right)$, the nodes in $O S^{f-2}$ are divided into two groups each of which is connected to $e_{1}$ and $e_{2}$, respectively.

\section{B. $2^{\text {nd }}$ Phase: Identifying Cave and forming a bi-connected topology}

Using the identified $d G$, OBiC opts to compute a concave hull of $S_{\text {seg }}$, Cave, based on which a Hamiltonian cycle of $S_{\text {seg }}$ is formed. Thus, the $2^{\text {nd }}$ phase is composed of two steps: computing Cave and forming a bi-connected undirected graph, $G_{2 c}$, of $S_{\text {seg }}$, by visiting once each of the internal segments, or internal nodes, in short iNodes, that reside in Cave.

Identifying the concave hull Cave: The idea of computing Cave is to form a mesh that involves the outmost segment group $O S^{0}$, the inmost one $O S^{f-1}$ and an internal bridge that connects $O S^{0}$ and $O S^{f-1}$ by visiting every $O S^{i}, 1 \leq i \leq f-2$ once, and which consequently covers the entire set of segments. The bridge is basically composed of two sub-paths that visit distinct segments between $O S^{0}$, and $O S^{f-1}$ and share no segments. In other words, each sub-path starts at $\mathrm{NiO}$ and 


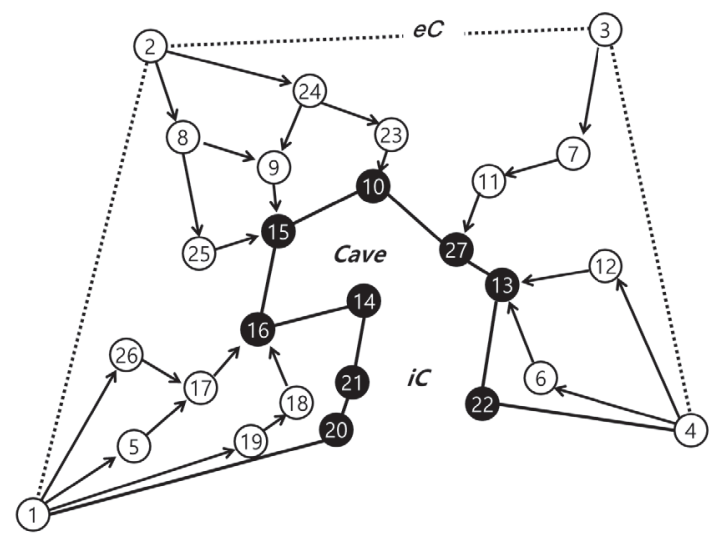

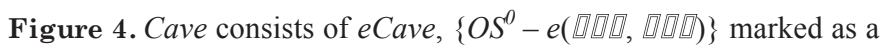
dotted outline and $i \operatorname{Cave}\left\{\left(\operatorname{OS}^{3}-e(\quad, \quad)\right) \mathbf{u}\right.$ bridge $\}$ marked as a bold line. The black nodes are already in a final Hamiltonian nislo

$N j O \in O S^{0}$ and ends at $N k f-1$ and $N l f-1 \in O S^{f-1}$ respectively, where $N i O \neq N j O, N k f-1 \neq N l f-1, \exists e(N i O$, $N j O) \in C H_{0}, \exists e(N k f-1, N l f-1) \in C H_{f-1}$, if $\left|O S^{f-1}\right| \geq 2$. As $\left|O S^{f-1}\right|$ is one, two sub-paths meet at one point $N k f-1(=N l f-1)$ in $O S^{f-1}$.

The goals for forming Cave based on a combination of $O S^{0}\left(\mathrm{CH}_{0}\right)$, and $\mathrm{OS}^{f-1}\left(\mathrm{CH}_{f-1}\right)$ are as follows: (i) making the formed Cave a basis through which iNodes inside Cave are biconnected; in order to achieve this goal, $\mathrm{OBiC}$ divides a layout of Cave into two sides; an external side of Cave, shortly eCave that includes the segments in $O S^{0}$ and an internal side of Cave, shortly $i$ Cave that combines $O S^{f-1}$ and the found bridge. Then $\mathrm{OBiC}$ ensures every segment in iNodes is connected to both sides using $d G$. (ii) making Cave vertex-disjoint; since $\mathrm{OBiC}$ tries to finally form a vertex-disjoint ring topology that is a Hamiltonian cycle, the identified Cave also ought to be nodedistinct. (iii) finding a cost-effective Cave with respect to the overall RN population for bi-connectivity; this goal is achieved by questioning what edge to pick on $O S^{0}$ to initiate the bridge formation. Since this edge is to be eventually replaced by the edges in the bridge, the longest edge is picked, which requires more RNs to be connected. Removing that edge will be consistent with the optimization objective of OBiC.

In order to identify such a node-distinct and cost-effective Cave, $\mathrm{OBiC}$ first initializes it as an undirected graph $G_{2 c}=\left(V_{2 c}\right.$, $E_{2 c}$ ) by adding $O S^{0}$ and $O S^{f-1}$, namely, $V_{2 c} \mathrm{U}=\{\forall i, j \mid \mathrm{NiO}$, $N j f-1\}, E_{2 c} \mathrm{U}=\left\{\forall i, j \mid \exists e(N i O, N j O) \in C H_{0}, \exists e(N i, N j)\right.$ $\left.\in \mathrm{CH}_{f-1}\right\}$. Then $\mathrm{OBiC}$ finds the two sub-paths of bridge by trying each edge in $\mathrm{CH}_{0}$ in non-decreasing order of length. Starting from the longest edge $e(\mathrm{NaO}, \mathrm{NzO}) \in \mathrm{CH}_{0}$, the two sub-paths of bridge are formed by finding two closest nodes $N a_{-}$1, and $N z_{-} 1$ in the inner group $O S^{l}$, from $\mathrm{NaO}$ and $\mathrm{Nz} \mathrm{O}$ respectively, where $\mathrm{Na} a_{-} 1 \neq \mathrm{Nz} z_{-1}$ and $N a_{-}{ }_{1}, N z_{-} 1$ is the smallest among $e(N a O, N i 1)$, and $e(N z O, N j 1), \forall i, j \in d E$. The edge selection is repeated in rounds by selecting the qualified nodes in $O S^{r} r=2, \ldots, f-1$. If $O S^{f-1}$ has only one node (segment), such a node is added in the internal bridge.

In case that the condition of the edge selection is not satisfied before reaching at $O S^{f-1}$, for example the selected two nodes in $O S^{r}$ are the same due to the nature of the found $d G$ based on the nested convex hull, the next longest edge in $O S^{0}$ is tried. The procedure is repeated until the edge selection condition is satisfactory up to $O S^{f-1}$. When the identification of bridge is done and Cave is completely formed by adding the edges between the selected nodes into $E_{2 c}$ and by removing $e(\mathrm{NaO}, \mathrm{NzO})$ in $O S^{0}$ and $e(N g f-1, N h f-1)$ in $O S^{f-1}$ only if $\left|O S^{f-1}\right| \geq 3$. For later use, the selected edges during forming bridge are removed from $d E$. After that the nodes on iCave except two end-nodes at which eCave merges, become a black node, which means it is already in a final Hamiltonian cycle.

Figure 4 shows the identified Cave based on the found $d G$ seen in Figure 2-(d). In the example, bridge is sought from two adjacent nodes in $O S^{0}$, i.e., $N 10$ and $N 40$ which forms the longest edge in $\mathrm{CH}_{0}$. Among the edges tailed at $\mathrm{N} 10$ and $N 40 \in d E$, the two edges whose head end-points are the closest, $e(N 10, N 201)$ and $e(N 40, N 221)$ are selected. Then the same procedure is repeated for N201 and N221, then $e(N 201, N 212)$ and $e(N 221, N 132)$ are selected. Finally, the found sub-paths, $\{e(N 10, N 201)$, $e(N 201, N 212), e(N 212, N 143)\}$ and $\{e(N 40, N 221)$, $e(N 221, N 132), e(N 132, N 123)\}$ are added into $E_{2 c}$ As a results, Cave is formed by combining eCave $=\left\{O S^{0}-\right.$ $e(N 10, N 40)\}$ and $i$ Cave $=\left\{\left(O S^{3}-e(N 143, N 123)\right) \cup\right.$ bridge marked as dotted lines and solid lines respectively as shown in Figure 4.

Bi-connecting iNodes: Throughout the identified Cave, $S_{\text {seg }}$ is bifurcated into a set of the on-segments lying on the perimeter of Cave and iNodes. Then OBiC opts to bi-connect iNodes via the on-segments and the found inward paths in $d G$. As seen in Figure 4 iNodes are grouped in multiple sub-digraphs, hereafter denoted as $d G(\mathrm{NiO})$, each of them has one of the onsegments of eCave, i.e., $\mathrm{NiO}$ in $\mathrm{OS}^{0}$ at a top of the sub-graph and reaches at some segments in $O S^{-1}$ at the bottom. In addition, the depth of all $d G(\mathrm{NiO})$ 's are the same for $\forall i$ as proven in Lemma 1.4. Then bi-connectivity of iNodes is performed per $d G(\mathrm{NiO})$, for which $\mathrm{OBiC}$ breaks a cycle in Cave by cutting its end-edge $e(S, E)$ of iCave that meets at eCave, hereafter denoted as Gate, inserts the vertex-disjoint path that spans from $S$ up to the deepest non-black iNodes $C$, and then encloses the loop by connecting $C$ to $E$. The same procedure is repeated for each of children of $S$ Children $(S)$ until all iNodes in $d G(\mathrm{NiO})$ are bi-connected via the extended Cave. More details will be explained in this sub-section. 


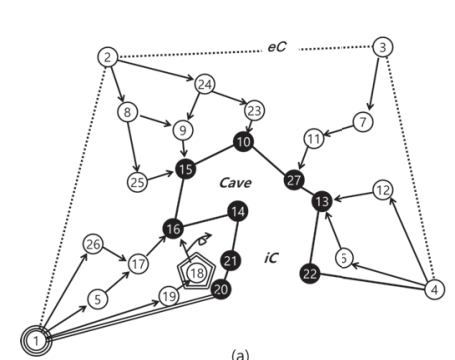

(a)

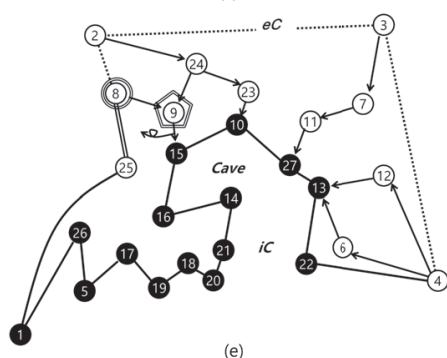

(e)
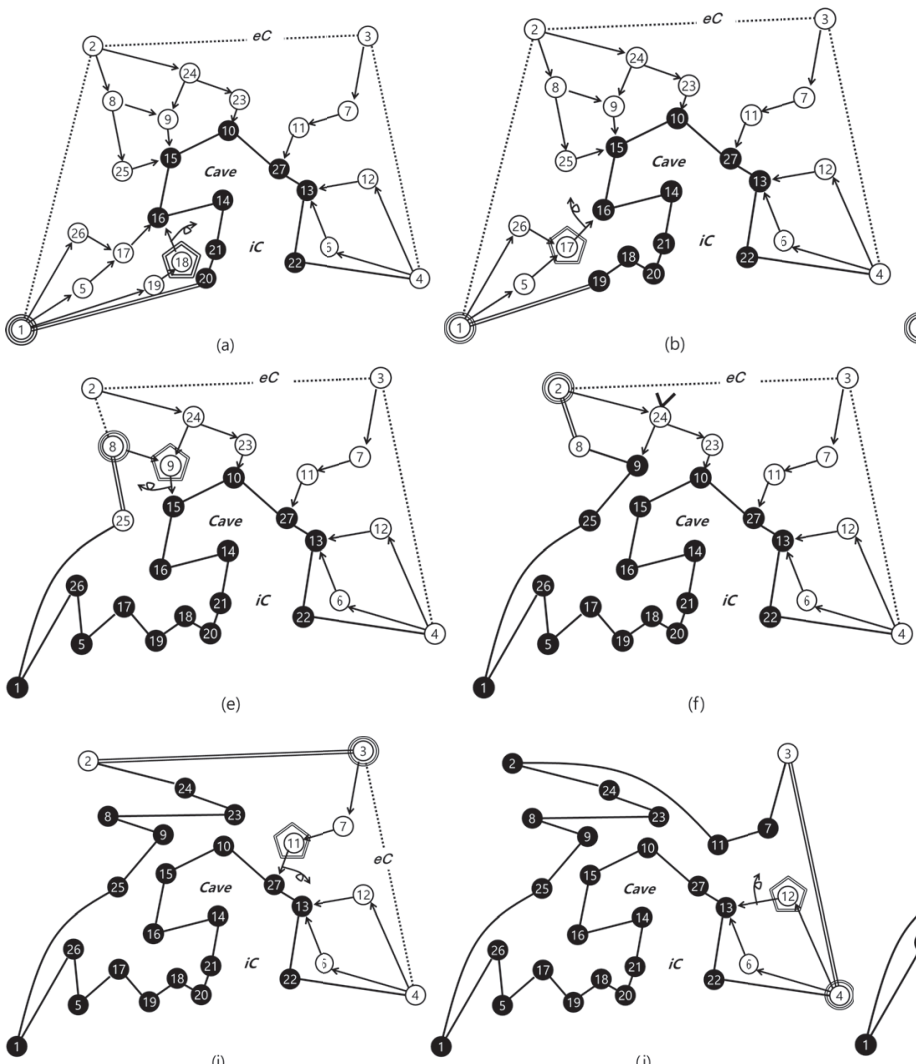

Figure 5. During the $2^{\text {nd }}$ phase, $\mathrm{OBiC}$ tries to bi-connect $i$ Nodes per $d G($ initial Cave gets more distorted and its size is thus minimized.

Bi-connectivity of iNodes begins at one of two merging points of eCave and iCave which are equivalent to two endnodes of the edge $e(\mathrm{NaO}, \mathrm{NzO})$ that has been removed from $O S^{0}$ when finding bridge. The selected point $\mathrm{NaO}$ or $\mathrm{Nz}$ Obecomes a ground point $S$, and the edge from $S$ to its black neighbor $S^{\prime}$ on iCave, e $\left(S, S^{\prime}\right) \in E_{2 c}$ becomes Gate. Then OBiC processes iNodes that belong to a sub-digraph rooted at $S$, i.e., $d G(S)$. In order to maintain an efficient Hamiltonian cycle during bi-connectivity of iNodes, OBiC processes Children(S) in non-decreasing order of perpendicular proximity to Gate from each of Children(S). Therefore, the nearest $i$ Nodes $x \in \operatorname{Children}(S)$ to Gate is first processed. For $x, \mathrm{OBiC}$ finds the vertex-disjoint path from $S$ to $i$ Cave. If there is only one path passing through $x$ and reaching at $i$ Cave, $\mathrm{OBiC}$ adds the path into $E_{2 c}$ and also includes an edge $e(L, S)$ in $E_{2 c}$, where $L$ is the last iNode in the path instead of removing the edge Gate from $E_{2 c}$. Then a closed loop is still maintained in Cave as an updated simple cycle which includes \{edges in the path that starts at $S$ and ends at $L$ \} U $\left\{e\left(L, S^{\prime}\right)\right\}$ instead of Gate $=e\left(S, S^{\prime}\right)$. After that iNodes along with the selected path, i.e., between $S$ and $S^{\prime}$ become black for meaning that they now join a Hamiltonian cycle. Otherwise in case that there exist multiple path of $S$ that goes through $x$, a sub-path between $S$ and $x$ is marked as eCave and, $x$ and $e\left(x, S^{\prime}\right)$

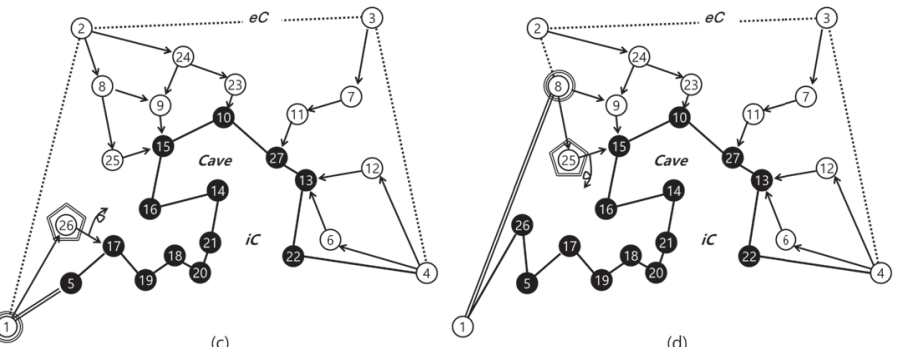

(c)

(d)
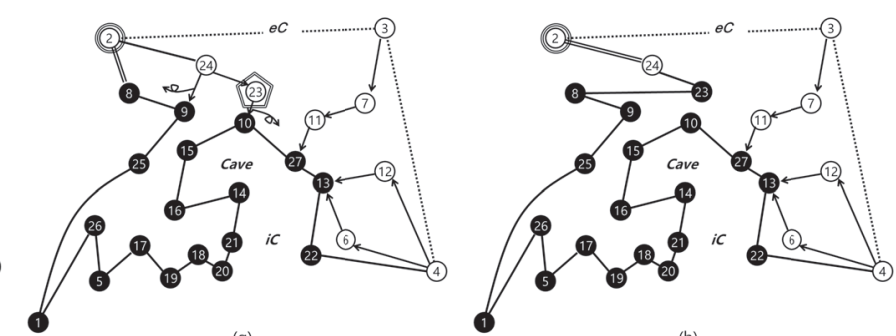

(g)

(h)

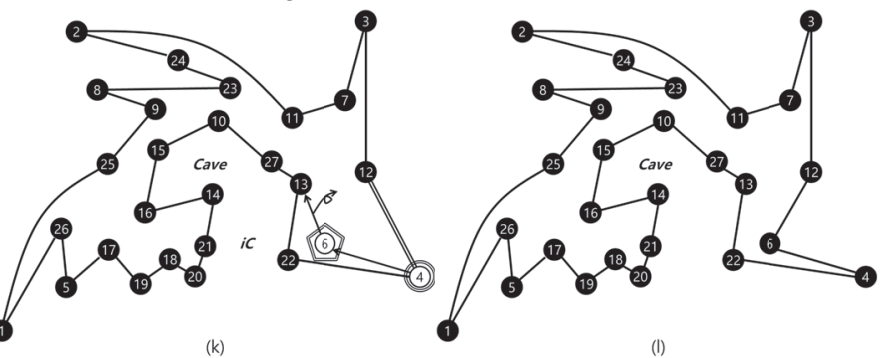

(l)

$0), i$, where $O$ is a node in $O S^{0}$. As the procedure progresses, the

become $S$ and Gate respectively. OBiC then restarts the biconnectivity procedure.

Each time when Cave is updated, Cave gets more distorted and its inner space is thus more minimized. The same process is repeated for another non-black child $x$ of $S$ which has not been processed if it exists. For the next round, the current $x$ and $e(S, x)$ becomes $S^{\prime}$ and Gate respectively. Bi-connectivity of $d G(S)$ is done when all iNodes in $d G(S)$ become black and $S$ also becomes a black node. After that a non-black neighbor of $S$ on $e$ Cave, says $S_{-}$becomes a new $S$ node and the old $S$ node switches to $S^{\prime}$. Then the same process is repeated for iNodes in $d G\left(S_{-}\right)$. In Figure 5 which shows an example for biconnectivity of iNodes, the procedure begins with $S=N 10, S^{\prime}$ $=N 201$ and Gate $=e(N 10, N 201)$. According to the nearest distance from Gate, $N 191, N 51$, and $N 71$ are processed in order. For $N 191(=x)$, a path for $N 10$ via $N 191$ is found and $\{N 191, N 181\}$ and $\{e(N 10, N 191), e(N 191, N 182)\}$ are added in $V_{2 c}$ and $E_{2 c}$ respectively. Also $e(N 182, N 201)$ is included in $E_{2 c}$ instead of Gate, e(N10,N201) removed from $E_{2 c}$. Finally, $N 191$ and $N 182$ become black as shown in Figure 5-(b).

Then in the next round where $N 191$ and $e(N 10, N 191)$ become $S^{\prime}$ and Gate respectively, $E_{2 c}$ is updated as $E_{2 c} \mathrm{U}=$ 
$\{e(N 10, N 51), e(N 51, N 172), e(N 172, N 191)\}-\{$ Gate $=e(N 10, N 191)\}$ and $N 51$ and $N 172$ become black as seen in Figure 5-(c). After the same procedure is repeated for $N 71$ and thus all $d G(N 10)$ turn black, another eCave node $N 20$ neighboring to $S(=N 10)$ becomes a new $S$ and then $d G(N 20)$ are processed for bi-connectivity. Figure 5-(d) shows that the case one of Children(N20), N81 $=x$ ) has more than one non-black child nodes N252 and N92, which means there are two separate paths from $N 20$ to iCave via $N 81$. Therefore, $d G(N 81)$ is processed prior to $d G(N 20)$. Figure 5-(1) shows the final Cave that forms a Hamiltonian cycle after processing $d G(N 30)$ and $d G(N 40)$,.

\section{C. $3^{\text {rd }}$ phase: Optimizing $G_{2 c}$ and populating $R N s$}

In the $3^{\text {rd }}$ phase, OBiC first strives to untangle the periphery of the polygon formed by $G_{2 c}$, via which all segments are biconnected during the $2^{\text {nd }}$ phase and then SPs where RNs would be placed, are identified on each edge in the updated $E_{2 c}$. The $3^{\text {rd }}$ phase thus consists of (i) disentanglement of edges, and (ii) edge steinerization for placing the least count of RNs.

Bi-connecting iNodes via the directed vertex-disjoint path may unnecessarily yield long edges in $E_{2 c}$ because the procedure is performed in rounds focusing on the formation of a cycle without considering the correlation of connectivity between the selected paths in adjacent rounds. For achieving the optimization objective of $\mathrm{OBiC}$, disentanglement of edges may thus be essential. The goal of the disentanglement is to reduce a sum of edge lengths, Length(e), $e \in E_{2 c}$ by eliminating zigzags so that the required number of RNs to biconnect all segments via $G_{2 c}$ is decreased. Let cost(path) denote a sum of the least count of RNs to connect individual links on that path. $\mathrm{OBiC}$ checks the entanglement per a tuple $T$ of three adjacent edges in $E_{2 c}$ for example $\left\{e_{I}(u, v), e_{2}(v, w)\right.$, $\left.e_{3}(w, x)\right\}$ by comparing $\operatorname{cost}\left(T^{\prime}\right)$ to that of an alternative $T^{\prime}$, i.e., $\left\{e_{1}^{\prime}(u, w), e_{2}^{\prime}(w, v), e_{3}^{\prime}(v, x)\right\}$. If $\operatorname{cost}\left(T^{\prime}\right)<\operatorname{cost}(T)$ then the edges in $T$ is replaced with the ones of $T^{\prime}$ in $E_{2 c}$. Since $G_{2 c}$ is the undirected graph, $e_{2}(v, w)$ actually equals $e_{2}^{\prime}(w, v)$. The disentanglement is performed in rounds until no more update occurs in $G_{2 c}$.

This procedure can be initiated at any segment in $V_{2 c}$, like $N 221$ seen in Figure 6-(a). Since for $T=\left\{e_{I}(N 221, N 40)\right.$, $\left.e_{2}(N 40, N 61), e_{3}(N 61, N 121)\right\}$ there is cost advantage in its $T^{\prime}$ which is $\left\{e_{1}^{\prime}(N 221, N 61), e_{2}^{\prime}(N 61, N 40), e_{3}^{\prime}(N 40\right.$, $N 121)\}$, change in $E_{2 c}$ is warranted. The same procedure is repeated for the other tuple such as $\left\{e_{I}(N 121, N 30)\right.$, $\left.e_{2}(N 30, N 71), e_{3}(N 71, N 112)\right\}$. Figure 6-(b) shows the same process is applied to three more tuples $\left\{e_{1}(N 71, N 30)\right.$, $\left.e_{2}(N 30, N 112), e_{3}(N 112, N 20)\right\},\left\{e_{1}(N 232, N 81)\right.$, $\left.e_{2}(N 81, N 92), e_{3}(N 92, N 252)\right\}$ and $\left\{e_{1}(N 252, N 10)\right.$, $\left.e_{2}(N 10, N 71), e_{3}(N 71, N 51)\right\}$. Then the $1^{\text {st }}$ round of disentanglement is done. In the $2^{\text {nd }}$ round, four more changes make $E_{2 c}$ updated as seen in Figure 6-(c) to (e) and then two more updates are applied to $E_{2 c}$ in the $3^{\text {rd }}$ round as seen in Figure 6-(f) and (g). Figure 6-(h) shows the final topology of $G_{2 c}$ when disentanglement terminates. 

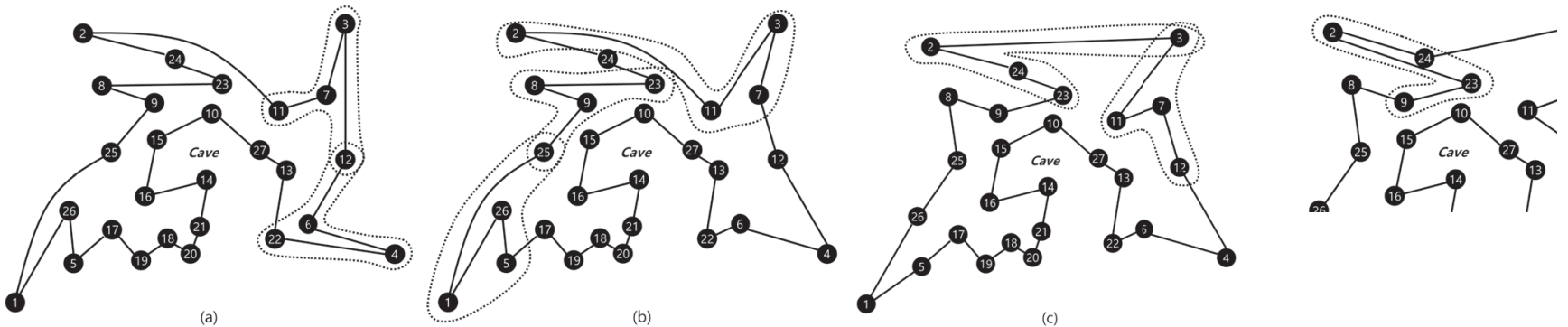

Figure 6. In the $3^{\text {rd }}$ phase, OBiC strives to reduce the circumference of $G_{2 c}$ by raveling the zigzags among the edges in $E_{2 c}$. For each tuple $T$ of three adjacent edges, OBiC compares a sum of the edge lengths in $T$ to that of an alternative $T^{\prime}$. When $\operatorname{cost}\left(T^{\prime}\right)<\cos t(T)$, the edges in $T^{\prime}$ replaces those of $T$ in $E_{2 c}$. The disentanglement is repeated in rounds until no change occurs. After 3 rounds, $G_{2 c}$ is finally updated into (h).

Based on the updated $G_{2 c}$, OBiC populates the least number of RNs to bi-connect the segments in $V_{2 c}$. Prior to the deployment of the least count of RNs, OBiC identifies a set of SPs at which RNs would be placed. During the identification of SPs, each edge $e$ in $E_{2 c}$ is steinerized while factoring in the communication range of a $\mathrm{RN}, R$, and a set of $\mathrm{SPs}, S_{S P}$, is found. The number of the identified SPs in $e,\left|S_{S P}(e)\right|$ equals $\lceil$ Length $(\mathrm{e}) / R\rceil-1$. Figure 7 shows the steinerized $G_{2 c}$ assuming $R$ is similar to the Euclidean distance between $N 92$ and $N 241$. Based on the found SPs, $G_{2 c}$ is updated to $G_{2 c}{ }^{\prime}=\left(V_{2 c}{ }^{\prime}\right.$, $\left.E_{2 c}{ }^{\prime}\right)$, where $V_{2 c}{ }^{\prime}=S_{\text {seg }} \cup\left\{S_{S P}(e), \forall e \in E_{2 c}\right\}$ and $E_{2 c}{ }^{\prime}=\{e \mid$ $\left.e(u, v), u, v \leq R, \forall u, v \in V_{2 c}{ }^{\prime}\right\}$. Then, $\mathrm{OBiC}$ populates a relay at $\forall v \in V_{2 c^{\prime}}$. The pseudo code of $\mathrm{OBiC}$ is described in Appendix B.

\section{Algorithm Analysis}

$\mathrm{OBiC}$ is analyzed in this subsection. We mainly focus on

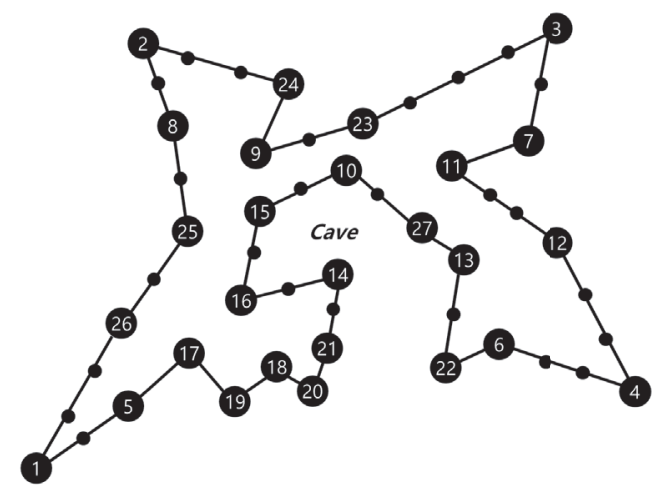

Figure 7. Each edge $e$ in $E_{2 c}$ is steinerized by a radio range of a RN $R$, assuming the $R$ is similar to the Euclidean distance between 92 and 241. Then the least count of RNs is populated on $e$. proving that $\mathrm{OBiC}$ converges to a bi-connected inter-segments topology which visits every segment at least once and thus the restored topology is fault-tolerant to a single node failure. In addition the complexity of $\mathrm{OBiC}$ is analyzed and it is proven that the resulting topology is formed within an execution time bound. We introduce the following theorems and lemmas:

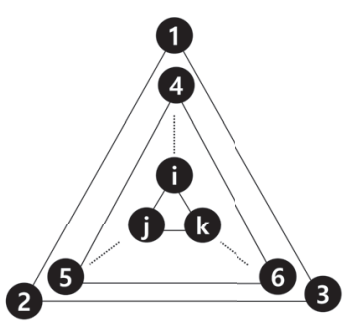

Figure 8. An example of the worst case of the $O S$ identification phase where

Lemma 1.1: The number of rounds $f$ of the OS identification of OBiC is bounded to $O([N \operatorname{seg} 37)$.

Proof: The categorization of $S$ seg during the first phase of $\mathrm{OBiC}$ is performed by computing a convex hull in rounds until the number of remaining segments is less than three and thus no more convex hull can be computed. Therefore, each $O S j$ except the final one should include at least three segments, i.e., $|O S j| \geq 3, \forall j$. In addition, the found $O S r-1$ in each round $r$ is obtained by computing a convex hull of the segments which have not been part of any of the previous $O S j$ 's, $\forall j<r-1$ and thus $O S O+O S 1+\ldots+O S f-1=j=O f-1 O S j$, where $f$ is a final round, should be equal to $\mid S$ seg $\mid$ also denoted as Nseg. Therefore, with a given $N$ seg a larger $f$ means fewer segments in each $O S j$. In other words, the least number of segments that is three in each $O S j$, makes $f$ the most. The worst case seen in 